\title{
Erratum to: Nonnegatively curved fixed point homogeneous 5-manifolds
}

\author{
Fernando Galaz-Garcia • Wolfgang Spindeler
}

Published online: 7 December 2013

C) Springer Science+Business Media Dordrecht 2013

\section{Erratum to: Ann Glob Anal Geom (2012) 41:253-263 DOI 10.1007/s10455-011-9282-0}

We fix a gap in the proof of the Main Theorem in the original article.

\section{Introduction}

We follow the same notation and numbering as in the original article. Lemma 3.3, originally stated in [1], was used in the proof of the Main Theorem in the original article. As stated, part (a) is incorrect, since the proof of this lemma requires the following assumption for (a) to hold: The preimage of $C^{*}$ is a smooth submanifold of $M$ without boundary. The correct statement of the lemma is therefore:

Lemma 3.3 Let $M^{n}$ be a closed, simply connected, nonnegatively curved manifold of dimension $n \geq 4$ with an isometric $\mathrm{S}^{1}$-action and suppose that $\operatorname{Fix}\left(M^{n}, \mathrm{~S}^{1}\right)$ contains an $(n-2)$ dimensional component $N$. Let $C^{*}$ be the set at maximal distance from $N^{*}$ in the orbit space $M^{*}$.

(a) If $\operatorname{dim} C^{*}=n-2$ and the preimage $C$ of $C^{*}$ is a smooth submanifold of $M$ without boundary, then $C$ is fixed by the $S^{1}$ action, $C^{*}=C$ is isometric to $N^{*}=N$ and is simply connected.

The online version of the original article can be found under doi:10.1007/s10455-011-9282-0.

F. Galaz-Garcia and W. Spindeler are part of SFB 878-Groups, Geometry and Actions at the University of Münster.

F. Galaz-Garcia $(\varangle) \cdot$ W. Spindeler

Mathematisches Institut, WWU Münster, Münster, Germany

e-mail: f.galaz-garcia@uni-muenster.de

W. Spindeler

e-mail: wolfgang.spindeler@uni-muenster.de 
(b) If $\operatorname{dim} C^{*} \leq n-4$, then $N^{*}$ is simply connected.

Lemma 3.3 was used in the proof of the following key observation in the original article to obtain a classification in the cohomogeneity 4 case:

Proposition 3.5 Let $M$ be a closed, simply connected nonnegatively curved 5-manifold with a fixed point homogenous $\mathrm{S}^{1}$-action and let $F$ be a three-dimensional component of $\operatorname{Fix}\left(M, S^{1}\right)$. Then there exists a closed, invariant and orientable smooth submanifold $H \subset M$ with $\operatorname{dim} H \in\{1,3\}$ such that $M$ is diffeomorphic to the union of two distance tubular neighbourhoods of $F$ and $H$ glued together along their common boundary E, i.e.,

$$
M=D(F) \cup_{E} D(H) .
$$

Without the additional assumption on Lemma 3.3 it was immediate to show that $\operatorname{dim} C^{*} \leq$ 2, implying $\operatorname{dim} H \leq 3$ (see the original article). Having in mind the corrected version of Lemma 3.3, a careful analysis when $\operatorname{dim} C^{*}=3$ gives rise to two cases that were not discussed in the original article. We analyze these two cases in the next section, fixing the gap in the proof of the Main Theorem in the original article.

\section{Missing cases}

To complete the proof of the Main Theorem in the original article, we prove Proposition 3.5 assuming $\operatorname{dim} C^{*}=3$ and using the corrected version of Lemma 3.3(a).

Recall that fixed point components of $S^{1}$, or of any subgroup of it, have even codimension, since $M$ is simply connected and hence orientable. First assume that $C^{*}$ contains some fixed point. Then, from Proposition 3.4 in the original article (whose proof does not use Lemma 3.3), we see that $C$ does contain a three-dimensional fixed point set component, say $F^{\prime}$. It easily follows that $C=F^{\prime}$ and we are done by setting $H=F^{\prime}$. Thus, we may assume that $C$ does not contain fixed points.

Case 1 Assume that $C^{*}$ contains only isolated exceptional orbits. Then we see from Proposition 3.4 of the original article that $C=\pi^{-1}\left(C^{*}\right)$ is a smooth submanifold, possibly with nonsmooth boundary. In fact, by Lemma 3.3, $C$ has nonempty boundary. Again by Proposition 3.4 of the original article, this is the case if and only if $C^{*}$ has a boundary component $B^{*}$ consisting exclusively of regular points. In this case, we let

$$
C_{2}^{*}=\left\{x \in C^{*} \mid d\left(x^{*}, B^{*}\right) \text { is maximal }\right\} .
$$

Following the arguments due to Grove and Searle [2], the distance function to $C_{2}^{*}$ is regular on $M^{*} \backslash\left\{C_{2}^{*} \cup F^{*}\right\}$ and the points in $C^{*} \backslash C_{2}^{*}$ correspond to principal orbits. This reduces the analysis to the case when $\operatorname{dim} C^{*}=2$, discussed in the original article.

Case 2 Suppose now that $C^{*}$ contains nonisolated exceptional orbits. In this case, there exists an isotropy group $\mathbb{Z}_{k}$, for some $k \geq 2$, with a three-dimensional fixed point component $B$. Observe that $B^{*}$ is two-dimensional and is contained in $\partial C^{*}$. Fix $p \in B$ and let $\mathrm{S}_{p}^{1}=\mathbb{Z}_{l}$, so $l \geq 2$ and $\mathbb{Z}_{k}$ is a subgroup of $\mathbb{Z}_{l}$. Let $v \in \mathbb{S}^{1} \subset T_{p} B^{\perp}$ such that $v^{*} \in v_{p^{*}} C^{*}$ (notice that $v_{p^{*}} C^{*}$ is nonempty, so such a $v$ does exist). The group $\mathbb{Z}_{l}$ acts with trivial isotropy at $v$ so its action on $\mathbb{S}^{1}$ is effective. Assume that $l \geq 3$. Then the set of vectors perpendicular to $\mathbb{Z}_{l}(v)$ is contained in $T_{p} B$, contradicting the fact that $C^{*}$ is three-dimensional. Thus $l=k=2$ and $\mathbb{Z}_{2}$ acts on $v_{p} B$ via reflection at 0 . Therefore, $B^{*}$ is a connected component of the nonregular part 
of $M^{*}$. We will now show that $B^{*}$ is also a connected component of $\partial C^{*}$. First note that $B^{*}$ is a closed subset of $\partial C^{*}$. Observe that the tangent cone $T_{p^{*}} C^{*}$ of $C^{*}$ at $p \in B^{*}$ is isometric to the half space $\mathbb{R}_{\geq 0} \oplus \mathbb{R}^{2}$, where the $\mathbb{R}^{2}$-factor corresponds to $T_{p^{*}} B^{*}$. By convexity $C^{*}$ is a three-dimensional Alexandrov space with boundary. By Perelman's conical neighborhood theorem, a sufficiently small neighborhood in $C^{*}$ of $p^{*}$ is homeomorphic to $T_{p^{*}} C^{*}$. Via such a homeomorphism it follows that $B^{*}$ is also open in $\partial C^{*}$. Therefore, $B^{*}$ is a boundary component of $C^{*}$.

Now, let us show that an open neighborhood of $B^{*}$ in $C^{*}$ lifts to a smooth submanifold of $M$ with empty boundary. Pick $p^{*} \in B^{*}$ and let $\exp _{p^{*}}$ denote the exponential map of $M^{*}$ at $p^{*}$. Again by convexity $\exp _{p^{*}}$ restricted to $T_{p^{*}} C^{*}$ coincides with the exponential map of $C^{*}$ at points where the latter is defined. Since there are no regular boundary points of $C^{*}$ near $B^{*}$ it follows that $\exp _{p^{*}}: T_{p^{*}} C^{*} \rightarrow C^{*}$ is defined for all $v \in T_{p^{*}} C^{*}$ with $\|v\|<\varepsilon$, for some $\varepsilon>0$. Consequently $B_{\epsilon}\left(p^{*}\right) \cap C=\exp _{p^{*}}\left(B_{\epsilon}(0) \cap T_{p^{*}} C^{*}\right)$. Now let $S$ be a slice at $p$. Consider the projections $\pi: M \rightarrow M^{*}$ and $h: T_{p} S \rightarrow T_{p^{*}} M^{*}$. Let $W=h^{-1}\left(T_{p^{*}} C^{*}\right) \subset T_{p} S$. By the discussion in the preceding lines, $W \cong \mathbb{R}^{3}$ is a linear subspace of $T_{p} S$ and $T_{p} M$. Then $N:=\exp _{p}\left(W \cap B_{\epsilon}(0)\right)$ is a smooth submanifold without boundary of $S$ and $M$, after choosing a smaller $\epsilon>0$ if necessary. We have

$$
\begin{aligned}
\pi(N) & =\pi\left(\exp _{p}\left(W \cap B_{\epsilon}(0)\right)\right) \\
& =\exp _{p^{*}}\left(h\left(W \cap B_{\epsilon}(0)\right)\right) \\
& =\exp _{p^{*}}\left(T_{p^{*}} C^{*} \cap B_{\epsilon}(0)\right) \\
& =B_{\varepsilon}\left(p^{*}\right) \cap C^{*} .
\end{aligned}
$$

Further, $S \cap \pi^{-1}(\pi(N))=N$ by the definition of $W$, therefore $S \cap \pi^{-1}\left(B_{\varepsilon}\left(p^{*}\right) \cap C^{*}\right)=N$. Now, $\pi^{-1}\left(B_{\varepsilon}\left(p^{*}\right) \cap C^{*}\right)=\mathrm{S}^{1}(N)$ is a smooth submanifold of $M$ without boundary.

From Proposition 3.4 of the original article we see that $\pi^{-1}(C)$ is a smooth submanifold of $M$, whose boundary components correspond precisely to regular boundary components of $C$. By Lemma 3.3 there exists such a boundary component. Proceeding as in Case 1, we reduce the analysis to the case when $\operatorname{dim} C^{*}=2$, discussed in the original article.

\section{References}

1. Galaz-Garcia, F., Searle C.: Nonnegatively curved 5-manifolds with almost maximal symmetry rank (2011, preprint). arXiv:1111.3183 [math.DG]

2. Grove, K., Searle, C.: Positively curved manifolds with maximal symmetry-rank. J. Pure Appl. Algebra 91(1-3), 137-142 (1994) 